OPEN ACCESS

Edited by:

Zuhua $\mathrm{He}$,

CAS Center for Excellence in Molecular Plant Sciences, China

Reviewed by:

Junzhong Liu,

Yunnan University, China

Yule Liu,

Tsinghua University, China

*Correspondence:

Yuriko Hayano-Saito

hsyuriko@affrc.go.jp

Specialty section:

This article was submitted to Plant Microbe Interactions,

a section of the journal

Frontiers in Plant Science

Received: 21 January 2020

Accepted: 06 April 2020

Published: 08 May 2020

Citation:

Hayano-Saito $Y$ and Hayashi $K$ (2020) Stvb-i, a Rice Gene Conferring Durable Resistance to Rice stripe virus, Protects Plant Growth From Heat Stress. Front. Plant Sci. 11:519. doi: $10.3389 /$ fpls.2020.00519

\section{Stvb-i, a Rice Gene Conferring Durable Resistance to Rice stripe virus, Protects Plant Growth From Heat Stress}

\author{
Yuriko Hayano-Saito* and Keiko Hayashi \\ NARO Central Region Agricultural Research Center, Tsukuba, Japan
}

Disease resistance is affected by temperature. A rice gene, Stvb-i, is known to have conferred sustained resistance to Rice stripe virus (RSV) despite global warming. Stvb$i$ protects plants from growth stunting caused by RSV. The underlying resistance mechanism is unclear. Here, Stvb-i showed stable RSV resistance for 20 years in laboratory experiments. This gene encodes a protein distinct from well-studied plant disease-resistance proteins. It has a domain homologous to the histidine kinase/heatshock protein 90-like ATPase superfamily. Rice has three paralogous genes including Stvb-i. The genes are expressed mainly in meristematic tissues. In the initial period after viral inoculation, RSV multiplication enhanced Stvb-i, whereas Stvb-i suppressed RSV multiplication. Stvb-i silencing inhibited plant growth regardless of viral infection, and silencing of the other paralogous gene that located closely to Stvb-i caused morphological abnormalities. The results suggested that the Stvb-i and its paralogs are related to plant development; especially, Stvb-i supports meristem growth, resulting in plant growth stabilizing. Growth stunting in the Stvb-i-silenced plants was more severe under repetitive heat stress, suggesting that Stvb-i contributed to the attenuation of heat damage in plant development. The symptoms of RSV infection (chlorosis, wilting, stunting, fewer tillers, and defective panicles) were similar to those of heat damage, suggesting that RSV multiplication induces heat-like stress in meristematic cells. Our findings suggest that the mechanism of meristem growth protection conferred by Stvb-i allows plants to withstand both heat stress and RSV multiplication. The suppression of RSV multiplication by the Stvb-i function in meristems results in durable resistance.

Keywords: Oryza sativa, virus resistance gene, Stvb-i, Rice stripe virus, durable resistance, heat stress, plant growth, developmental homeostasis

\section{INTRODUCTION}

Plants need to withstand abiotic and biotic stresses such as temperature fluctuations, water scarcity (drought), wind, excessive or insufficient light, and pathogens. Viral multiplication in plant meristems directly threatens plant survival because meristems are indispensable for plant growth and development. Plants have evolved sophisticated systems that allow them to avoid or reduce damage to meristems. 
Many rice viruses inhibit plant growth (Ling, 1972; Hibino, 1996; Satoh et al., 2010, 2011, 2013; Budot et al., 2014). In particular, Rice stripe virus (RSV), an RNA virus causative of rice stripe disease, invades plants when they are sucked by the small brown planthopper (SBPH, Laodelphax striatellus Fallén) and greatly damages rice production, mainly in temperate East Asia, the world leading rice production area (Hibino, 1996; Otuka, 2013). In RSV-infected plants, typical leaf symptoms are discontinuous pale-yellow stripes, chlorotic streaks, and wilting (Ling, 1972). Systemic symptoms include stunted plant growth, fewer tillers, and defects in panicle formation and grain filling (Ling, 1972; IRRI Rice Knowledge Bank ${ }^{1}$ ). Infection at the seedling stage causes severe leaf and systemic symptoms, resulting in plant death (Ling, 1972; Hibino, 1996). Serious damage is supposed to be determined by where RSV multiplies and how it spreads. After invasion, RSV immediately migrates to meristematic tissue at the base of the seedling, which provides cells for the basic structure of the plant body, multiplies there and spreads systemically with active cell division (Sonku and Sakurai, 1973). RSV particles were observed in cells of the meristems including apical domes and leaf primordia (Takahashi et al., 2008).

An RSV resistance gene, $S t v b$ - $i$, has been introgressed into Oryza sativa spp. japonica cultivars from the indica cultivar Modan (Hayano-Saito et al., 1998, 2000). The Stvb-i-harboring rice seedlings show milder symptoms than susceptible rice: faint discontinuous leaf stripes, reduced systemic symptoms, and decreased death by wilting (Washio et al., 1967, 1968). Stvb-i does not completely suppress RSV multiplication, and RSV is detectable at a low level in seedling base tissues including the meristem (Hayano-Saito, 2014).

Abiotic stresses influence plant resistance to viruses; some resistance genes are temperature sensitive (Wang et al., 2009; Kobayashi et al., 2014). A major type of plant resistance genes encodes nucleotide-binding site and leucine-rich-repeat (NBSLRR) domain proteins, which elicit a hypersensitive response (Wang et al., 2009; Kobayashi et al., 2014). The genes for NBSLRR proteins lose their function above a certain temperature (Samuel, 1931; Wang et al., 2009; Kobayashi et al., 2014). RSV multiplication is easily enhanced by short high-temperature treatment $\left(42^{\circ} \mathrm{C}\right.$ for $30 \mathrm{~min}$ ) in Nicotiana benthamiana (Jiang et al., 2014). However, the hypersensitive response is not observed in Stvb-i-harboring plants (Hayano-Saito et al., 2000), and the effect of temperature has not been investigated.

Viral resistance is often rapidly weakened or overcome (Kobayashi et al., 2014). Interestingly, Stvb-i-harboring rice varieties have shown stable resistance for over 50 years in the paddy fields of Japan (Hayano-Saito, 2014). Stvb-i is located in the multi-allelic Stvb locus (Washio et al., 1968), and several resistant alleles have been reported (Hayano-Saito et al., 2000; Maeda et al., 2006; Wang et al., 2011; Wu et al., 2011; Zhang et al., 2011; Kwon et al., 2012). Among the allelic genes, STV11 derived from an indica rice Kasalath has been isolated (Wang et al., 2014) but the relationships among the allelic genes remain unclear. To elucidate the durability of RSV resistance mediated

${ }^{1}$ http://www.knowledgebank.irri.org/ by $S t v b-i$, we investigated the role of this gene in rice and its effects on RSV multiplication. We revealed the structure of the $S t v b-i$ gene and found that it functions in developmental homeostasis, especially in meristematic tissues, contributing to the recovery from heat stress. Heat stress alleviation mediated by $S t v b-i$ reduces RSV multiplication as well as leaf and systemic symptoms. Protection of meristems from stresses confers durable RSV resistance to St $v b$ - $i$-harboring plants. Our study helps to understand the intricate relationships between a plant and its pathogenic virus and provides important information on stress tolerance in meristems.

\section{MATERIALS AND METHODS}

\section{Plant Materials}

Rice (O. sativa) cultivars Tsukinohikari, Asanohikari, and Musashikogane are RSV-resistant descendants of a resistant cultivar St No. 1 into which the RSV resistance gene Stvb-i of the indica rice cultivar Modan was introgressed; all three cultivars have the Stvb-i gene (Hayano-Saito et al., 1998; HayanoSaito, 2002). Two RSV-susceptible japonica paddy cultivars, Nipponbare and Koganebare, were used as control cultivars in inoculation tests. Nipponbare is the standard rice cultivar used in the International Rice Genome Sequencing project. Koganebare is a progeny of Nipponbare; their properties are very similar, including stripe disease susceptibility (Koumura et al., 1980). Koshihikari, Kirara397, and Yuukara are also RSVsusceptible japonica paddy cultivars. Recombinant inbred lines (3,629 lines) at the $\mathrm{F}_{7}$ generation developed from a cross between Tsukinohikari and Koganebare were used to delimit the Stvb$i$ region; among them, RIL3245 was resistant and RIL484 was susceptible (Hayano-Saito, 2002). Yuukara and RIL484 were used in the complementation test. St No. 1, Tsukinohikari and Yuukara were used in the RNAi-mediated suppression test.

\section{Inoculation With RSV and Assessment of Resistance}

To evaluate RSV resistance, bioassays using viruliferous SBPHs in the seedling test (Washio et al., 1967, 1968) were conducted as described elsewhere (Hayano-Saito et al., 1998). The susceptible cultivars were Nipponbare, Koganebare, Koshihikari, Kirara397, and Yuukara; resistant cultivars were St No. 1, Tsukinohikari, Asanohikari, and Musashikogane. A colony of the vector (infective ability $>60 \%$ ) derived from viruliferous SBPHs collected in the Kanto district (Japan) was obtained from the former National Agriculture Research Center (currently called the Central Region Agriculture Research Center, Tsukuba, Japan) in 1990. To maintain the high infective ability of the colony, RSV-infective SBPHs were reselected two or three times a year by inoculation tests or a serological method (Washio et al., 1968). The SBPH population used in the bioassays recirculated the virus between rice and the insects. RSVinoculated plants were grown in a glasshouse and plants with symptoms were counted 4-5 weeks after inoculation. Bioassays were carried out in 1990-1992 at Tsukuba (National Institute of Agrobiological Science, $36^{\circ} 03^{\prime} 10.6^{\prime \prime} \mathrm{N}, 140^{\circ} 10^{\prime} 15.2^{\prime \prime} \mathrm{E}$ ) and 
1996-2009 at Sapporo (Hokkaido Agricultural Research Center, $43^{\circ} 00^{\prime} 92.8^{\prime \prime} \mathrm{N}, 141^{\circ} 41^{\prime} 15.0^{\prime \prime} \mathrm{E}$ ) in Japan. Weather data for Tsukuba were obtained from the weather data acquisition system of the Institute for Agro-Environmental Sciences ${ }^{2}$ and the data for Sapporo were obtained from the meteorological observation system at the National Agricultural Research Center for Hokkaido Region since $1990^{3}$.

The ratio of diseased plants represents the ratio of the percentage of diseased plants in the tested population to that in the population of Nipponbare (control). Each plant with chlorosis (Supplementary Figure S1A) was counted as diseased. Reactions to RSV were evaluated by the "ratio of disease-rating index" (RDRI) as described elsewhere (Washio et al., 1967, 1968) (Supplementary Figure S1B). Based on the RDRI, the cultivars and lines were classified as resistant $(\mathrm{R}, \mathrm{RDRI} \leq 30)$, moderately resistant $(\mathrm{M}, 30<\mathrm{RDRI} \leq 60)$, or susceptible $(\mathrm{S}$, RDRI > 60). The RDRI data were obtained from six replications of the complementation test and from three replications of the RNAi-mediated suppression test. Thirty plants were used in one replication.

Rice stripe virus inoculation for tissue staining and RNA analysis was performed as follows: rice seedlings at the 1.0-1.5leaf stage were exposed to 10 viruliferous SBPHs per plant at $25-27^{\circ} \mathrm{C}$ in a growth chamber, and the insects were removed after $24 \mathrm{~h}$. Non-viruliferous SBPHs were used for mock-inoculation of control plants. Plants were kept in the growth chamber until RNA preparation.

\section{Immunohistochemistry}

Paraffin sections were prepared from 3-5 $\mathrm{mm}$ pieces of the bases of rice seedlings. Seedling bases at 5-10 days after RSV inoculation were sampled. Sections were stained with a Vectastain ABC-AP kit (Vector Laboratories ${ }^{4}$ ) and antibody against RSV (rabbit IgG, Japan Plant Protection Association), and were observed under a digital microscope VHX-5000 (Keyence ${ }^{5}$ ) using without imaging modification.

\section{Sequencing, Gene Annotation, and Sequence Similarity Search}

An ABI310 genetic analyzer and a BigDye Terminator Cycle Sequencing Kit version 3.1 (Applied Biosystems ${ }^{6}$ ) were used for DNA sequencing. The Stvb-i genomic region sequences of St No. 1 and Nipponbare provided by the Rice Genome Project (National Institute of Agrobiological Sciences, Tsukuba, Japan) were used for marker development and sequence comparison. Candidate $S t v b$ - $i$ ORFs were predicted and annotated with the Rice Genome Automated Annotation System (Sakata et al., 2002). Protein domain analysis of Stvbi was performed with InterPro $^{7}$. Paralogous gene information was obtained from the

\footnotetext{
${ }^{2}$ http://www.naro.affrc.go.jp/org/niaes/aws/

${ }^{3}$ http://www.naro.affrc.go.jp/harc/kisyo/index.html

${ }^{4}$ https://vectorlabs.com

${ }^{5}$ https://www.keyence.com

${ }^{6}$ https://www.thermofisher.com/jp/ja/home.html

${ }^{7}$ http://www.ebi.ac.uk/interpro/
}

MSU Rice Genome Annotation Project ${ }^{8}$ (Kawahara et al., 2013). The sequences of Stvbi paralogs were aligned by using GenetyxMac (ver. 18, Genetyx ${ }^{9}$ ).

\section{Genotyping Analysis}

Cleaved amplified polymorphic sequence, simple sequence repeat, and simple sequence length polymorphism markers were newly developed from the comparison between the genomic sequences of St No. 1 and Nipponbare (Supplementary Table S1). These PCR-based markers and restriction fragment length polymorphic markers (Hayano-Saito, 2002) were used for fine genotyping analyses of recombinant inbred lines. DNA extraction and genotyping analyses were performed as described elsewhere (Hayano-Saito et al., 1998; Saito et al., 2010).

\section{DNA Constructs and Rice Transformation}

The DNA of the $S t v b-i$ region covered by the BAC clone St0014J19 (Supplementary Figure S2) was purified using a Large-Construct Kit $\left(\right.$ Qiagen $\left.^{10}\right)$. Genomic DNA of St No. 1 was purified as described elsewhere (Hayano-Saito et al., 1998), partially digested with Sau3AI, and size-fractionated in the $20-25-\mathrm{kb}$ range by ultra-centrifugation (5-25\% stepwise gradient of $\mathrm{NaCl}$ containing $3 \mathrm{mM}$ EDTA, 200,000 $\times g$, $4.5 \mathrm{~h}$ at $20^{\circ} \mathrm{C}$, Hitachi RPS40T rotor). A transformationcompetent artificial chromosome (TAC) library was constructed in BamHI-digested pYLTAC7 as described elsewhere (Liu et al., 1999). Clones carrying candidate ORFs were selected from the library and introduced into ElectroMAX Agrobacterium tumefaciens LBA4404 (Invitrogen ${ }^{11}$ ). Plants were transformed with A. tumefaciens as described elsewhere (Ozawa, 2009). Transgenic lines with the StD fragment (accession no. LC157868) evaluated for RSV resistance in the complementation test were derived from RIL484 (DR201, DR202, and DR204), and Yuukara (DY401, DY402, DY505, DY508, and DY510).

For RNAi-mediated suppression of ST07i (the candidate for $S t v b-i)$, an internal fragment, NK5 trigger $(370 \mathrm{bp}$, Supplementary Figure S3), was amplified with the NK5 primer set (Supplementary Table S1) and was used as an inverted repeat to transcribe the trigger dsRNA. The fragment was inserted into the pANDA vector as described elsewhere (Miki and Shimamoto, 2004). The RNAi construct was introduced into St No. 1, Tsukinohikari, and Yuukara as described above. The NK5-RNAisilenced lines St507 and St509 were derived from St No. 1; Tu518 and Tu520 from Tsukinohikari; and Yu5001, Yu5002, and Yu5008 from Yuukara. Four transgenic lines (St507, St509, Tu518, and Tu520) were used for RNAi-mediated suppression tests; St507, St509, Yu5001, Yu5002, and Yu5008 were used for gene expression analyses and plant phenotyping. For stable stress tolerance evaluation, we used $\mathrm{T}_{6}$ generation transgenic lines

\footnotetext{
${ }^{8}$ http://rice.plantbiology.msu.edu/

${ }^{9}$ https://www.genetyx.co.jp

${ }^{10}$ https://www.qiagen.com/us/

${ }^{11}$ https://www.thermofisher.com/jp/ja/home.html
} 
with fixed plant properties, instead of unstable $T_{2}$ generation transgenic lines.

\section{RNA Preparation}

Total RNA was extracted by using TRIZOL Reagent or TRIZOL Plus RNA Purification Kit (Invitrogen) from 3-5-mm pieces of the bases of seedlings (including leaf and root primordia and shoot apices, Figure 1A) and tillers (including leaf primordia and shoot apices), 5-mm pieces of the 3rd leaf blades of seedlings, 10$\mathrm{mm}$ pieces of flag leaf blades, 10 - $\mathrm{mm}$-long young whole panicles (including multiple primordia in each glumous flower) in the sheath, and $100 \mathrm{mg}$ of callus prepared for transformation as above. Etiolated seedlings were grown at $25-27^{\circ} \mathrm{C}$ in a plant growth chamber in the dark. Seedling bases were sampled from six plants and other parts from three plants.

\section{Cloning of Stvb-i and Its Paralogs}

For Stvb-i cloning, total RNA was extracted from 10 seedlings. The $5^{\prime}$ end of the transcript was identified using $5^{\prime}$-rapid amplification of cDNA ends (RACE) Core Set (Takara-Bio ${ }^{12}$ ). Full-length cDNA was generated using an RNA LA PCR Kit (Takara-Bio), cloned using a Mighty TA-Cloning Kit (TakaraBio), and sequenced. The transcripts of Stvb-i paralogous genes were identified on the basis of database information, amplified from suitable tissues as above and sequenced.

\section{Expression Analyses of Viral and Rice Genes}

All primers and the sizes of the amplified fragments are listed in Supplementary Table S2. To analyze the expression of rice and viral genes, reverse transcription-polymerase chain reaction (RT-PCR) was carried out using an RNA-PCR Kit (Takara-Bio). The RSV genome encodes the following proteins: RdRp, RNAdependent RNA polymerase; P2, membrane-associated protein; Pc2, membrane glycoprotein; NS3, non-structural protein 3; CP, coat protein; P4, major non-structural protein; and Pc4, nonstructural protein 4 (Zhu et al., 1991, 1992; Takahashi et al., 1993; Toriyama et al., 1994). The primers for RSV genes were developed using the following sequences: accession nos. D31879.1 (RdRp on RNA1), D13176.1 (P2 and Pc2 on RNA2), D01094.1 (NS3 and $C P$ on RNA3), and D01039.1 (P4 and Pc4 on RNA4). Rice glyceraldehyde 3-phosphate dehydrogenase (OsGAPDH, accession no. AK064960) was used as an internal reference gene.

To measure $S t v b$ - $i$ expression in RSV-infected plants, seedlings were sampled every day at noon. Total RNA was prepared from six seedlings inoculated with or without RSV. Quantitative RTPCR was conducted on an ABI7500 Real-time PCR System (Applied Biosystems) using a SYBR PrimeScript RT-PCR Kit (Takara-Bio). Stvb-i expression levels were determined by the standard curve method; rice OsGAPDH (see above) was used as an internal reference gene. To compare gene expression in heated and non-heated rice plants, total RNA was prepared from four seedlings per treatment. Quantitative RT-PCR was conducted on a Roche Lightcycler 480 Real-Time PCR system (Nippon

\footnotetext{
${ }^{12} \mathrm{https}: / /$ www.takara-bio.com
}

Genetics ${ }^{13}$ ) using Thunderbird SYBR qPCR Mix $\left(\right.$ Toyobo $\left.^{14}\right)$. The results were analyzed by the $\triangle \triangle \mathrm{CT}$ method; rice OsGAPDH was used as an internal reference gene. The mean relative expression levels and the SEM $(n=3)$ were calculated using the statistical package BellCurve for Excel (Social Survey Research Information ${ }^{15}$ ). A Tukey-Kramer multiple comparison test was used for data analysis.

\section{Plant Height and Tiller Number}

Two Stvb-i-silenced lines (St507 and St509) and three Stvb-icomplemented lines (Yu5001, Yu5002, and Yu5008) were used for plant phenotyping as follows.

To investigate the relationship between plant growth and RSV inoculation, St No. 1 and Stvb-i-silenced lines (15 plants per line; 15 plants per dish of $13 \mathrm{~cm}$ in diameter and $3 \mathrm{~cm}$ in depth) were used. Seedlings were inoculated with RSV at 1.5-leaf stage ( 6 days after germination) and the aboveground height was measured at 36 days after germination. Susceptible Nipponbare was used as reference.

For tiller counting, Stvb-i-silenced and -complemented lines of St No. 1 and Yuukara (15 plants per line; 3 plants per 15$\mathrm{cm}$ diameter pot) were used. Non-infected plants were grown under normal growth conditions from May to August in Tsukuba, Japan. The tillers were counted 54 days after germination (vegetative stage) and 84 days after germination (heading stage).

To investigate the role of $S t v b-i$ in heat stress, $S t v b-i$-silenced lines and St No. 1 were used. The aboveground height of 20 RSV-uninfected plants was measured from day 0 (start) to day 10. Leaf stage was measured from day 0 to day 11 with reference to Hoshikawa (1989).

All data were analyzed by a one-way or two-way analysis of variance (ANOVA), and if necessary a Tukey-Kramer multiple comparison test.

\section{Heat Treatment of Rice Seedlings}

Healthy seeds with coats removed were surface-sterilized with sodium hypochlorite solution $(2.5 \%$ effective chlorine concentration), washed several times with sterile distilled water, and soaked in sterile distilled water at $20^{\circ} \mathrm{C}$ for germination. Germinated seeds were planted in soil and seedlings were grown at $25^{\circ} \mathrm{C}(16 \mathrm{~h}$, light $)$ and $20^{\circ} \mathrm{C}(8 \mathrm{~h}$, dark $)$ in a controlled plant growth chamber. Rice seedling growth declines above $35^{\circ} \mathrm{C}$ (Krishnan et al., 2011). For observation of plant appearance during the whole growth period, $S t v b$-i-silenced and -complemented plants were exposed at $35-37^{\circ} \mathrm{C}$ for 4 days at 1 stleaf stage (4 days after germination) and were grown in a plant growth chamber for 7 days and then in a glasshouse. Similar observations were performed three times. To investigate the role of $S t v b-i$ in heat stress, 20 seedlings of each of the silenced lines and WT St No. 1 at the 1st-leaf stage were left untreated or were heat-treated at $38^{\circ} \mathrm{C}$ for 2 days once or three times, and then grown at $25^{\circ} \mathrm{C}(16 \mathrm{~h}$, light $)$ and $20^{\circ} \mathrm{C}(8 \mathrm{~h}$, dark $)$ in a

\footnotetext{
${ }^{13} \mathrm{https} / / /$ www.n-genetics.com

${ }^{14} \mathrm{https} / / /$ www.toyobo-global.com

${ }^{15} \mathrm{https} / / /$ www.ssri.com
} 


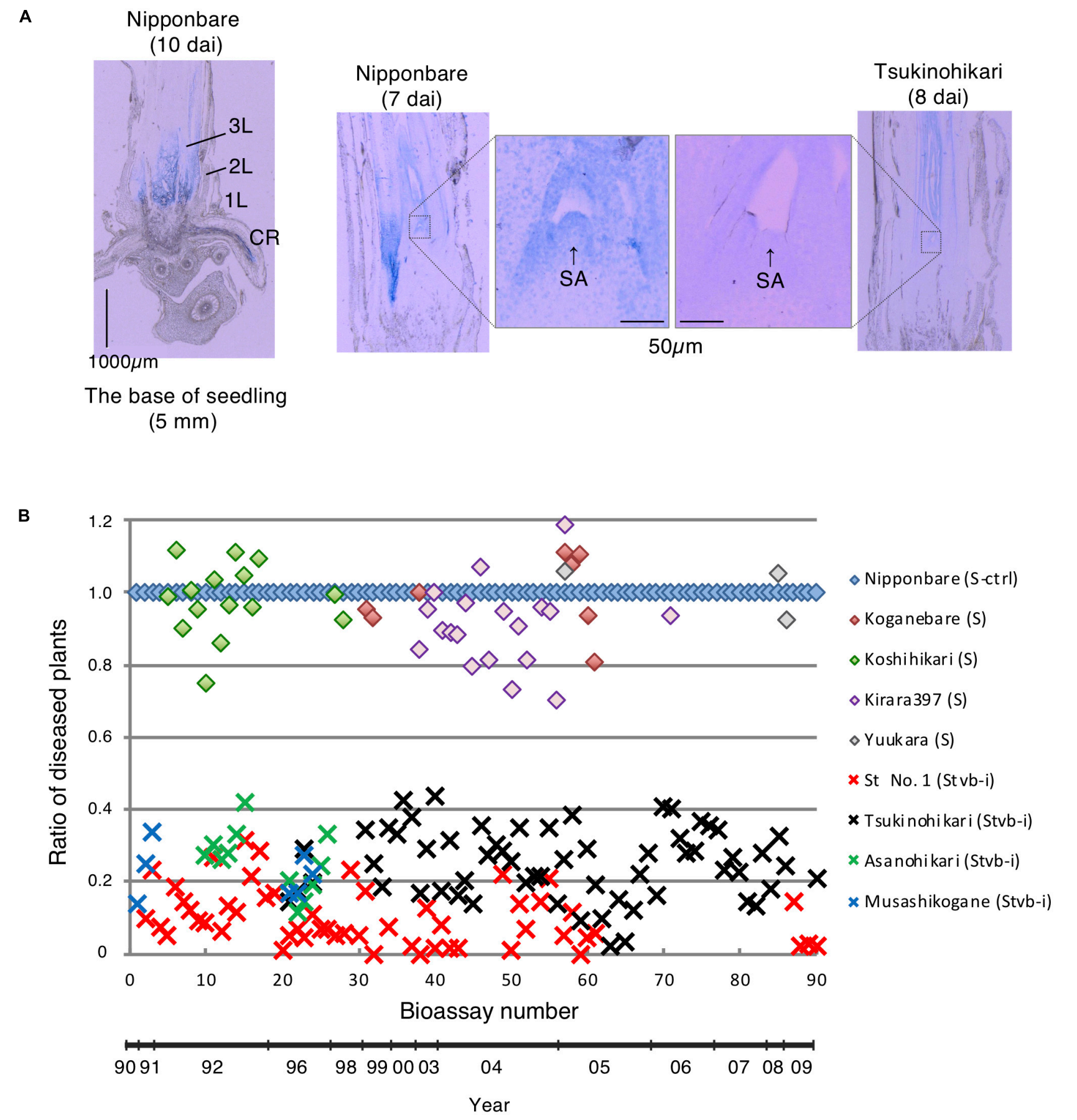

FIGURE 1 | (A) Viral localization in meristematic issue by immunostaining. 1L, 1st leaf; 2L, 2nd leaf; 3L, 3rd leaf; CR, crown root; SA, shoot apex. (B) Bioassays for rice stripe virus resistance conferred by Stvb-i from 1990 to 2009. Reaction to the virus is presented as the ratio of diseased plants. The ratio of diseased plants represents the ratio of the percentage of diseased plants in the tested population to that in the population of Nipponbare. In each bioassay, 30-180 plants per cultivar were tested. Nipponbare was used as a susceptible control cultivar (S-ctrl).

controlled plant growth chamber. The plant height and leaf stage were determined (see section "Plant Height and Tiller Number").

For expression analysis, Stvb-i-silenced lines and St No. 1 were used. Germinated seeds were placed on $0.4 \%$ gellan gum containing 1/5 Murashige-Skoog medium in a plastic box for plant tissue culture. The seedlings were heat treated at $38^{\circ} \mathrm{C}$ for $12 \mathrm{~h}$ at the 1.5-leaf stage. Meristematic tissues were sampled at 0 , $6,12,18,24,36,48$, and $60 \mathrm{~h}$ from the start of heat treatment (at 09:00 h), and RNA preparation and analysis were performed as described above. 


\section{RESULTS}

\section{RSV Localization and Durability of Stvb-i-Mediated Resistance}

In an RSV-resistant cultivar Tsukinohikari at 10 days after RSV inoculation, viral concentration at the seedling base is low and viral spread to the upper leaves is suppressed as compared to a susceptible cultivar Nipponbare (Hayano-Saito, 2014). We used an immunohistochemical technique to examine the localization of RSV in the base tissue of rice plants (Figure 1A). In Nipponbare, RSV spread mainly in the upper part of the 1st node at 10 days after RSV inoculation; the signals were especially dense in the primordium and shoot apex at 7 days after RSV inoculation. This indicates that RSV is present and propagates in meristems, and then spreads to other plant tissues and organs. In Tsukinohikari harboring $S t v b-i$, the signals were strongest in the upper part of the developing leaves in the sheath, were weaker in the base part of the leaves, and were very weak in the meristem. These data suggest that, although RSV multiplies in young leaves, the increase in the number of virus particles is suppressed in the meristem of Tsukinohikari, i.e., Stvb-i-mediated resistance suppresses RSV propagation in meristematic tissue.

We evaluated the severity of RSV infection on the basis of leaf symptoms, mainly chlorosis (yellowing) and twisting (Supplementary Figure S1A), in four resistant cultivars (St No. 1, Tsukinohikari, Asanohikari, and Musashikogane) and five susceptible cultivars (Nipponbare, Koganebare, Koshihikari, Kirara397, and Yuukara) in 90 bioassays performed over 20 years (Figure 1B). In bioassays, we used the SBPH population recirculated the virus between rice and the insects.

The ratio of diseased plants was considerably lower in the presence of $S t v b-i$; in all resistant rice cultivars, it rarely exceeded 0.4 (Figure 1B). The resistance of St No. 1 was slightly stronger than those of its progeny, Tsukinohikari, Asanohikari, and Musashikogane. The pathogenicity of RSV did not change over the 20 years. These observations indicate that $S t v b-i$-mediated resistance is durable.

\section{Isolation of the Stvb-i Gene}

We isolated $S t v b-i$ by map-based cloning. Genetic analysis using recombinant inbred lines delimited the Stvb-i locus to a 48 $\mathrm{kb}$ interval between markers ST49 and ST82 on the long arm of chromosome 11 (Figure 2A and Supplementary Figure S2). We compared this region with the genome of the susceptible cultivar Nipponbare. The Stvb-i-harboring RSV-resistant cultivar St No. 1 had a Rim2/Hipa transposon (Wang et al., 2003). A 10,136-bp segment was substituted by a 6,383-bp segment, with low sequence identity between the two segments. Mapping identified eight putative genes (ST01-ST08) as candidates for $S t v b-i$ (Figure 2A). Each candidate gene region alone failed to confer RSV resistance, however, introduction of the StD fragment (accession no. LC157868; Figure 2A) conferred resistance in susceptible RIL484 and Yuukara (Figure 2B).

The StD fragment covered the coding regions of three predicted candidate genes, ST05, ST06, and ST07. Using RACE analyses, we found another candidate gene, ST07i, in the StD fragment (Figure 2A). Suppression of ST07i expression by RNA silencing made the transgenic lines of resistant cultivars susceptible to RSV (St507, St509 in the St No. 1 background; Tu518, Tu520 in the Tsukinohikari background; Figure 2C). St507 and St509 tended to be higher RDRI values than did Tu518 and Tu520 (Figure 2C). Thus, the results of bioassays (Figures 1B, 2C) suggest that the genetic background affects RSV resistance level.

Thus, we concluded that ST07i is Stvb-i (Figure 2D). This gene located at LOC_Os11g31480 spans $11.2 \mathrm{~kb}$, has five exons and encodes a 1,649-aa protein that has no NBS-LRR domain but has a domain homologous to the histidine kinase/HSP90like ATPase superfamily (IPR036890). The result indicates that Stvb-i differs from the RSV-resistance gene STV11 (OsSOT1, LOC_Os11g30310), which is derived from an indica rice Kasalath and encodes an enzyme sulfotransferase (Wang et al., 2014).

\section{Viral RNA Production and Stvb-i Expression}

Three days after inoculation, propagation of RSV particles in seedling base tissues including the meristem is higher in susceptible rice than in resistant rice (Hayano-Saito, 2014). The abundance of viral RNA species encoded both in the viral sense (RdRp, $P c 2, C P$, and $P c 4)$ and complementary sense (P2, NS3, and $P 4$ ) increased more slowly in a resistant cultivar than in a susceptible cultivar (Figure 3A). The viral sense RNAs were detected clearly later (by 3 days for $R d R p$ and $P c 2$; by 1 day for $C P$ and $P c 4$ ) in the resistant cultivar than in the susceptible cultivar. Thus, although RSV is able to multiply in both resistant and susceptible rice cultivars, multiplication is slower in resistant rice. This result is consistent with the immunohistochemical observation (Figure 1A).

The delay of RSV RNA replication in the resistant cultivar Tsukinohikari occurred within a few days after viral inoculation. We examined $S t v b-i$ expression in the initial 0-5 days after RSV inoculation in Tsukinohikari (Figure 3B). Stvb-i was expressed before RSV inoculation (at day 0) (Figure 3B). In non-inoculated seedlings, the level of $S t v b-i$ mRNA gradually increased from day 2 to day 5 to twice the initial level. In contrast, it increased sharply on day 3 upon RSV inoculation and then plateaued; the level on day 5 was similar in non-inoculated and inoculated seedlings. Stv $b-i$ upregulation on day 3 was too late to halt viral RNA replication because RSV had already started multiplying by this time (Figure 3A). However, it might be sufficient to suppress further RSV multiplication.

We next investigated $S t v b-i$ expression in the silenced lines derived from St No. 1, in which the effect of $S t v b$ - $i$ was slightly stronger than in Tsukinohikari (Figures 1B, 2C). In the Stvb$i$-silenced lines St507 and St509, Stvb-i expression was almost undetectable before RSV inoculation and for up to 5 days after inoculation, but increased slightly 5-10 days after RSV inoculation (Figure 3C), probably because of the presence of the viral RNA-silencing suppressor NS3 (Xiong et al., 2009). In spite of restored $S t v b-i$ expression, the infected plants were severely damaged (Figure 2C). This result indicates that resistance is not 
A Stvb-i Centromere

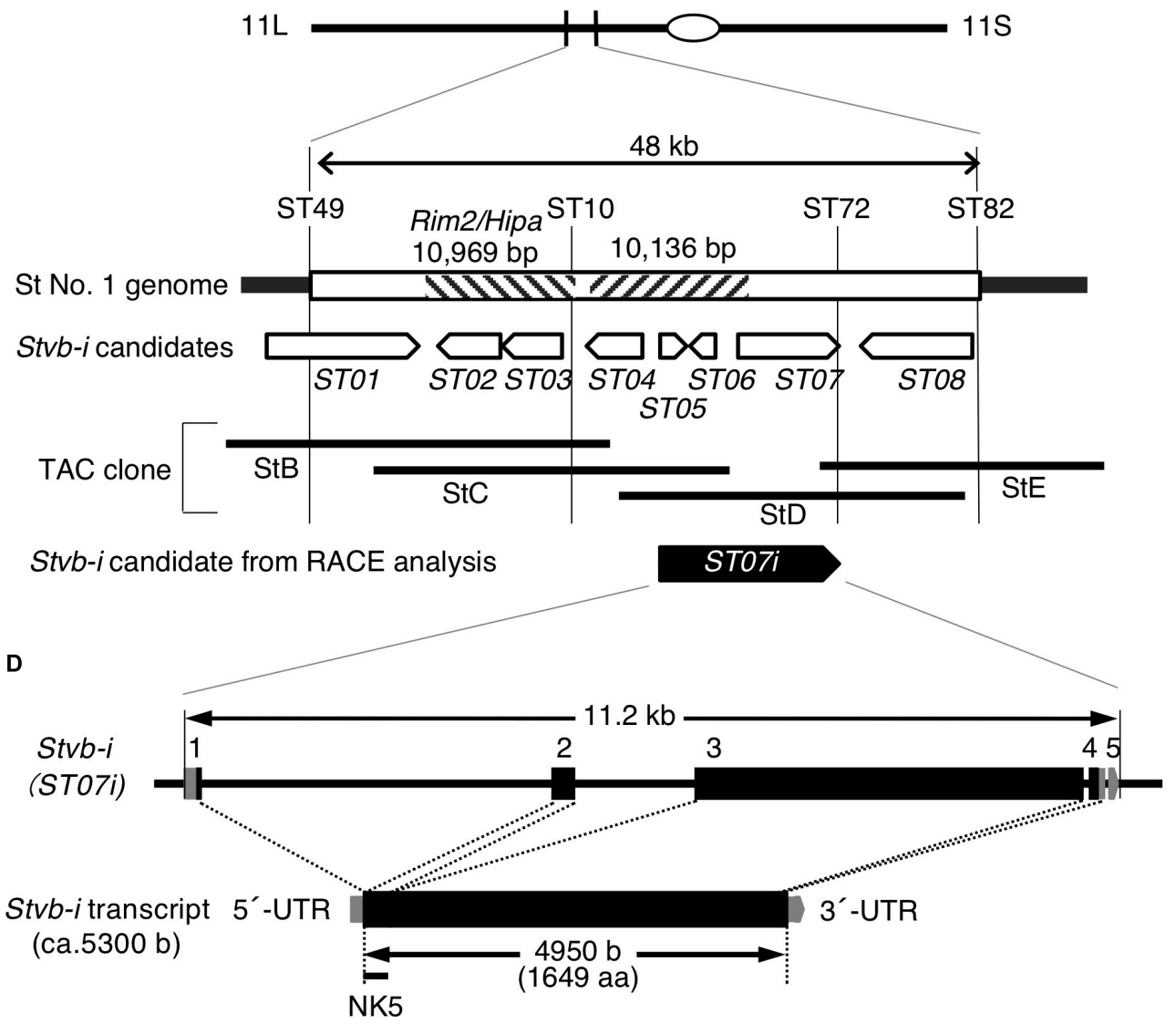

B

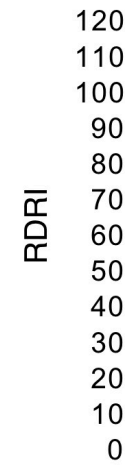

C

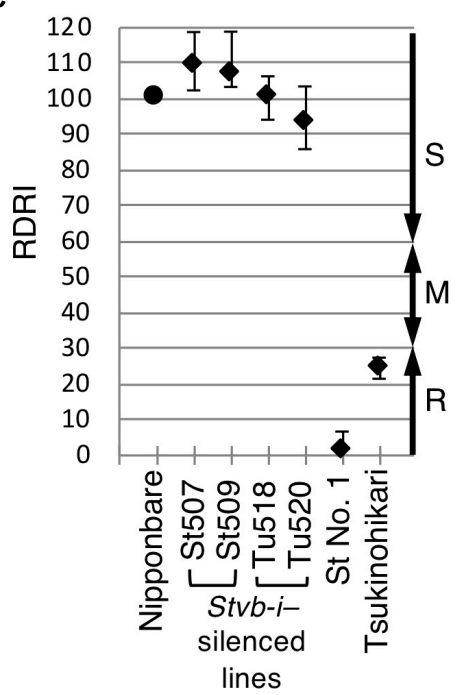

FIGURE 2 | Map-based cloning of the Stvb-i gene. (A) Fine physical map of the 48-kb Stvb-i region, structure of the Stvb-i locus, and the Stvb-i candidate genes. Pentagons represent the predicted candidate genes and the identified candidate gene, STO7i, and their direction. A Rim2/Hipa transposon is present in St No. 1 but not in Nipponbare. In the Nipponbare genome, a 10,136-bp segment is substituted by a 6,383-bp segment with low sequence identity. (B) Average disease rating indexes of StD lines relative to that of Nipponbare (RDRI) ( $n=6$; capped bars show the ranges). Control cultivars were Nipponbare (susceptible) and St No. 1 (resistant). (C) Average RDRIs of RNAi lines $(n=3)$ compared with Nipponbare and St No. 1. (D) Structure of Stvb-i. Coding regions are shown in black and the untranscribed regions (UTRs) in grays. NK5 represents a trigger fragment for the RNAi construct. 


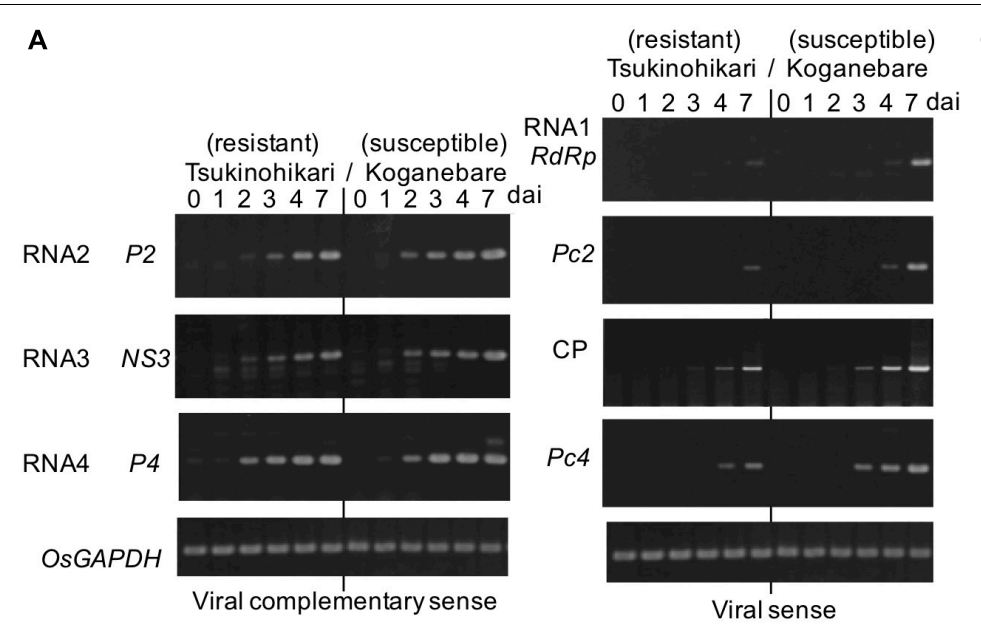

C
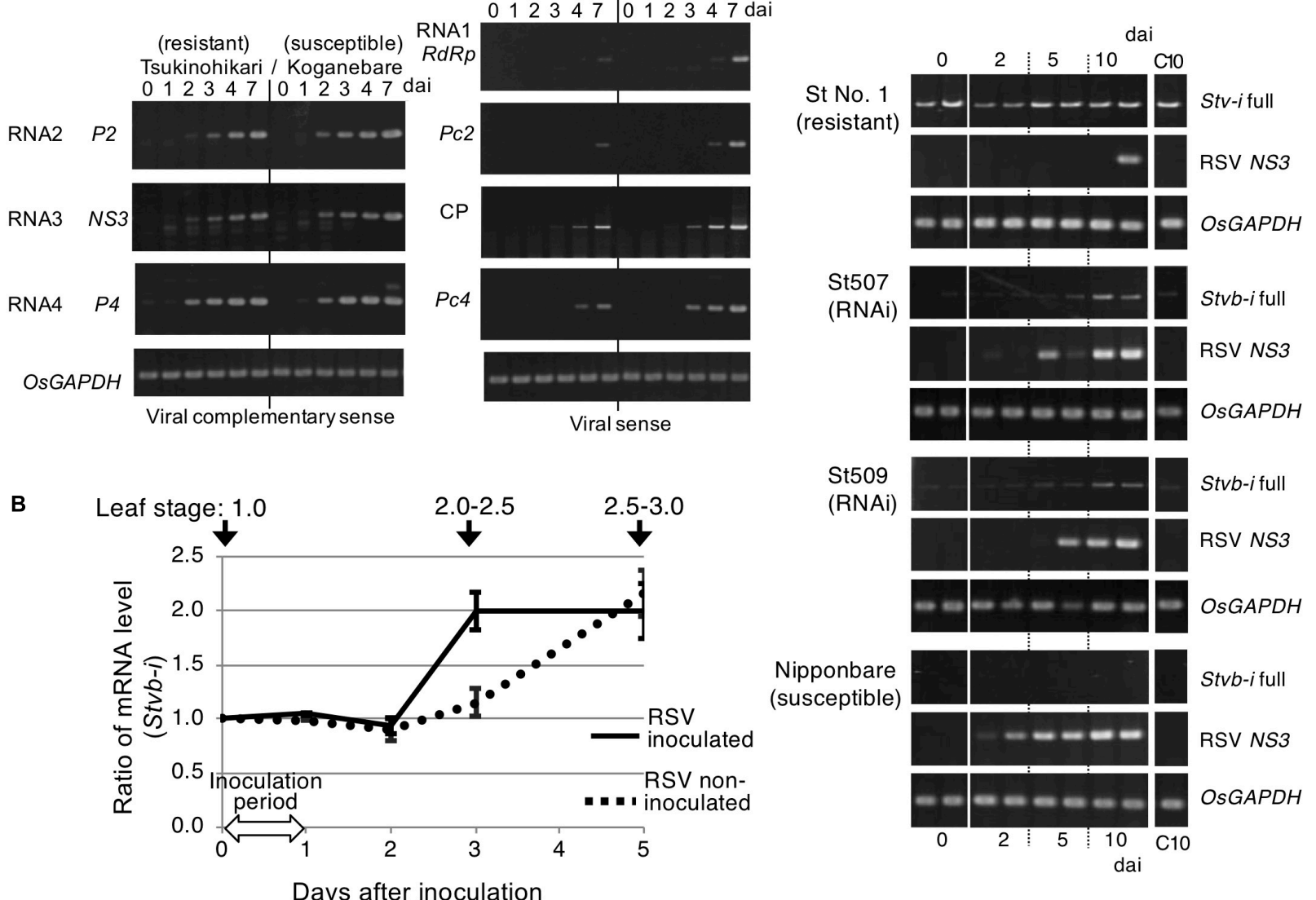

FIGURE 3 | Expression of RSV genes and Stvb-i in seedling meristems. (A) Expression patterns of all seven genes present in the RSV genome (RdRp, P2, PC2, NS3, CP, P4, and PC4) in the cultivars Tsukinohikari (resistant) and Koganebare (susceptible). dai, days after inoculation. (B) Quantitative RT-PCR analysis of Stvb-i expression in Tsukinohikari. Transcript levels are shown relative to the level on day 0 (start of RSV inoculation), and are means \pm SEM ( $n=3$ ). (C) Expression of Stvb-i and RSV NS3 in the Stvb-i-silenced transgenic T2 lines St507 and St509. C10, non-inoculated plants of the same type at 10 dai were used as controls.

affected by restoration of $S t v b-i$ expression after 5 days postinoculation.

Taken together, the results suggest that Stvbi presence in the seedling meristem prior to RSV invasion is required for RSV resistance and that $S t v b-i$ is upregulated in response to RSV multiplication.

\section{Genes Paralogous to Stvb-i}

While comparing the sequences of the $S t v b-i$ region on rice chromosome 11 between St No. 1 and Nipponbare, we found two paralogous genes in each cultivar: ST01 and Stvb-i in St No. 1, and ST01-allelic Os11g31500 and Stvb-i-allelic stvb$j$ (Os11g31480) in Nipponbare (Figure 4A). The amino acid sequence similarity among the four deduced proteins exceeded 90\%. A BLAST search identified an additional Stvbi paralog encoded by Os12g29350 on chromosome 12 of Nipponbare (Figure 4A). All five paralogs had an N-terminal domain homologous to that in histidine kinase/Hsp90-like ATPase superfamily proteins, implying that these proteins have the same or similar functions.
The Stvb-i-paralogous genes showed different expression patterns in plant tissues (Figure 4B). In resistant St No. 1, Stvb$i$ was expressed in plant parts containing meristems (seedling base, axillary tiller base, and 10-mm-long young panicle), and was highly expressed in calluses, but not in elongated leaf blades (Figure 4B and Supplementary Figure S4). The $s t v b-j$ gene of susceptible Nipponbare was expressed only in young panicles. ST01 of St No. 1 and Os11g31500 of Nipponbare were expressed mainly in young panicles. Os11g31500 was also expressed at a low level in the seedling base, but ST01 was not. Os12g29350 was expressed in all tested tissues of both St No. 1 and Nipponbare; the expression was stronger in immature plant parts containing meristems than in mature tissues such as leaf blades (Figure $4 \mathbf{B}$ ). Only $S t v b-i$ was expressed specifically in seedling and tiller meristems, where RSV multiplies.

\section{Role of Stvb-i and Paralogous Genes Phenotypes of Stvb-i-Silenced Lines}

Rice stripe virus infection caused more severe stunting in the RNAi lines than in WT St No. 1, and both lines were significantly 


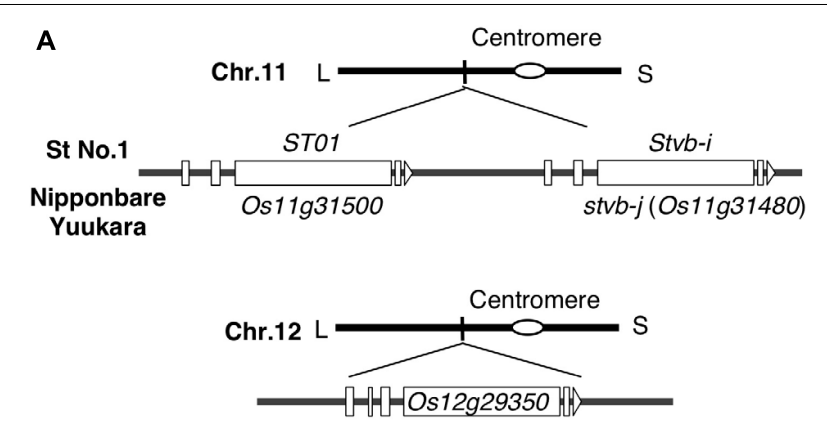

B

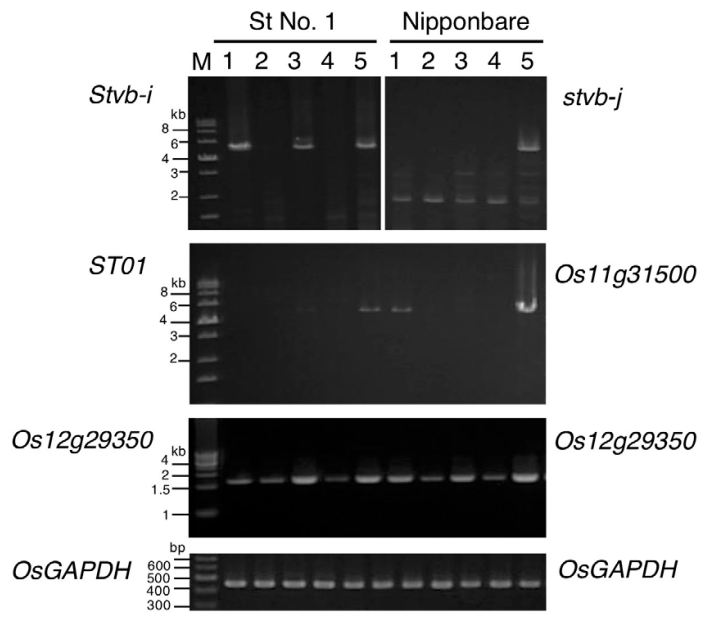

FIGURE 4 | Stvb-i paralogous genes and their expression. (A) The structures of the Stvb-i paralogs STO1, stvb-j (Os11g31480), Os11g31500, and Os12g29350. These four genes are shown according to sequence information in the database of the MSU Rice Genome Annotation Project (http://rice.plantbiology.msu.edu). L, long arm; S, short arm. (B) Expression of Stvb-i paralogs. Lanes 1, seedling bases; 2, mature 3rd-leaf blades; 3, tiller bases; 4, mature flag-leaf blades; 5 , young panicle (10 mm in length); $\mathrm{M}$, molecular DNA size marker: Gene Ladder 100 (0.1-2 kbp, Nippongene) for OsGAPDH and Perfect DNA Markers (0.5-12 kbp, Novagen) for the others.

stunted regardless of RSV (Figure 5A). There was an interaction between RSV infection and rice cultivar or line in plant height ( $d f=3, M S=154.03, F=9.720, P=0.000$ in two-way ANOVA). Non-inoculated mature plants of these lines also had fewer tillers (Figure 5B) and showed drying of mature (elongated) leaves and bending of the lamina joint (Supplementary Figure S5A). These results suggest that $S t v b-i$ has an important role in rice development.

\section{Phenotypes of Os11g31500-Suppressed Lines}

We examined the phenotypes of Os11g31500-suppressed lines, Yu5001, Yu5002, and Yu5008. The expression of the paralogous gene Os11g31500 was suppressed by RNAi generated using the NK5 construct (Supplementary Figures S3, S6), which suggests that Os11g31500 is a functional analog of Stvb-i in the susceptible cultivar Yuukara. Although plant height was not significantly affected in the RNAi-suppression lines (data not shown), the tiller number was decreased significantly in all
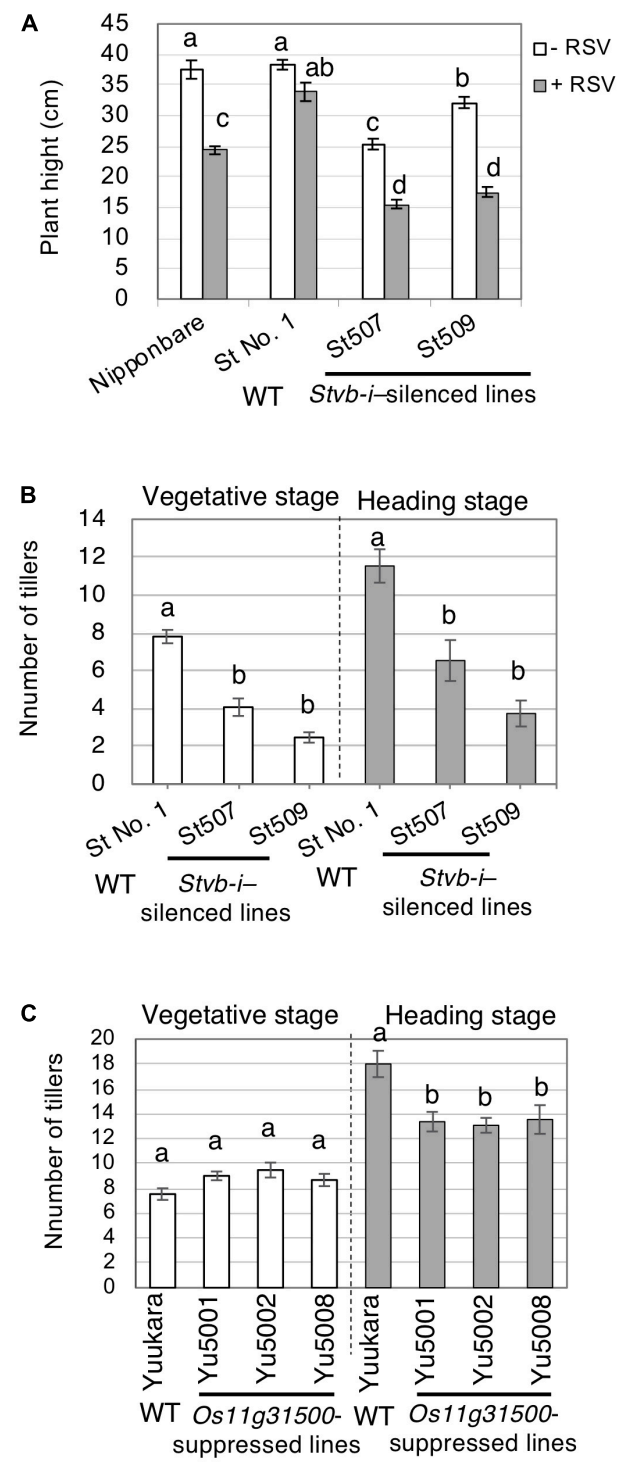

FIGURE 5 | Role of Stvb-i and Stvb-i paralogous genes in plant growth. (A) Height of aboveground parts of rice plants at 36 days after planting. (B) Average number of tillers in non-inoculated plants of Stvb-i-silenced lines at the vegetative and heading stages. (C) The average number of tillers at the vegetative and heading stages in three non-inoculated RNAi lines (Os11g31500-suppressed lines), Yu5001, Yu5002, and Yu5008. In all three panels, different letters indicate significant difference (Tukey-Kramer test, $p<0.01, n=15)$. WT, wild type.

three lines at the heading stage, but not at the vegetative stage (Figure 5C). Long upper glumes were frequently observed in the Os11g31500-suppressed lines (Supplementary Figure S7). Together with the expression pattern of Os11g31500 (Figure 4B), these results suggest that $O s 11 g 31500$ is primarily involved in rice development at the reproductive stage.

\section{Role of Stvb-i in Heat Stress}

The Stvb-i-silenced plants seemed to be affected by temperature: their growth was suppressed more strongly in hot summers 
at Tsukuba in $2015\left(36^{\circ} 03^{\prime} \mathrm{N}\right.$; maximum temperature, $30.2^{\circ} \mathrm{C}$ in July and $30.4^{\circ} \mathrm{C}$ in August) than in mild summers at Sapporo in $2009\left(43^{\circ} 00^{\prime} \mathrm{N}\right.$; maximum temperature, $21.8^{\circ} \mathrm{C}$ in July and $24.1^{\circ} \mathrm{C}$ in August); leaf drying, chlorosis and lamina joint bending were observed at both locations, and panicle malformation was observed at Tsukuba in the Stvb-i-silenced line (Supplementary Figure S5). We hypothesized that the effect of $S t v b-i$ silencing on plant growth is particularly strong under heat stress.

Heat treatment (4 days at $35-37^{\circ} \mathrm{C}$ at the 1 st-leaf stage) promptly caused chlorosis on whole seedling and short crown root (Figure 6A). Five days after the end of treatment, yellowing of leaves and slowed root development were alleviated, and the difference in plant phenotypes was unobservable between Stvb$i$-silenced lines (St507 and St509) and the wild-type St No. 1 (Figure 6B). However, the silenced lines showed stunted growth up to 28 days after the end of the heat treatment in comparison with the wild type and non-heated plants, which recovered within an overall shorter period (Figure 6B). At 56 days after the heat treatment, the silenced lines were still stunted, whereas the treated and un-treated WT plants grew similar to each other (Figure 6B). The same heat stress experiment was performed on the $S t v b-i$-complemented lines (DY505 and DY508). In contrast to the $S t v b$-i-silenced lines, the complemented lines grew without stunting (Figure 6B). The silenced plants could not recover from heat damage suffered at the seedling stage, suggesting a positive effect of Stvb-i on growth of heated seedlings.

We exposed 1st-leaf-stage seedlings of the silenced lines to $38^{\circ} \mathrm{C}$ for 2 days once or three times and examined the height of the seedlings in detail. Heat treatment accelerated the leaf stage progress (Figure 6C), but there was no significant difference in leaf stage at day 11 between the silenced lines and the WT in each treatment (Supplementary Table S3). In the silenced lines, stunting appeared immediately after the heat treatment, whereas non-heated seedlings grew similarly with or without $S t v b-i$ up to 3-leaf age (Figure 6C). The growth difference between the Stvb$i$-silenced lines and the WT increased with each exposure to high temperature (Figure 6C). The results indicate that the presence of $S t v b-i$ attenuates the damage caused by heat stress and that $S t v b-i$ relates in regulation of heat-responsive pathway; this gene contributes to plant growth.

\section{Heat Response of Stvb-i and Hsp70 Genes}

Heat shock proteins 70 (Hsp70s) are important in the thermal response (Wang et al., 2004; Wahid et al., 2007), and are involved in viral infection and multiplication (Mayer, 2005; Nagy et al., 2011). We examined the expression of St $v b-i$ and four rice $H s p 70$ genes in heated plants using quantitative RT-PCR. The transcripts of three Hsp70s-Os03g60620, Os11g08470, and Os05g38530accumulate at 30 days after RSV inoculation (Jiang et al., 2014), and $\mathrm{Os} 05 \mathrm{~g} 38530$ is reportedly upregulated by heat stress $\left(42^{\circ} \mathrm{C}\right.$ $10 \mathrm{~h})$ (Hu et al., 2009). Another Hsp70, Os11g47760, encodes a protein highly homologous to Os03g60620, Os05g38530, and NbHsp70 (KX912913) of N. benthamiana. NbHsp70 is associated with multiplication of Chinese wheat mosaic furovirus in $N$. benthamiana and affects leaf development (Yang et al., 2017).

In the WT St No. 1, the Stvb-i expression level seemed to increase slightly in heated than in unheated plants and was about twice the level of unheated plants at $6 \mathrm{~h}$; the expression levels became similar at later time points regardless of heat treatment (Figure 7). Silencing of Stvb-i significantly increased the expression level of $0 s 03 g 60620$ at several time points and tended to slightly increase the expression level of Os11g47760 at all time points (Supplementary Figure S8).

In $S t v b-i$ silenced lines, unlike in WT, heat greatly decreased the expression of Os03g60620 at 6-24 h (Figure 7). On the other hand, heat had no effect on the expression of $O s 05 g 38530$ and Os11g047760 at $6 \mathrm{~h}$, and on Os11g08470 expression at $12 \mathrm{~h}$ (Figure 7). The results suggest that $S t v b-i$ involves in heatresponsive control of the Hsp70 genes, especially Os03g60620.

\section{DISCUSSION}

In response to various external stimuli and environmental stresses, plants modulate various signaling pathways in meristems that support plant development. In this study, we found that the RSV resistance gene $S t v b-i$ in rice contributes to developmental homeostasis in meristems and focused on the role of this gene in resistance to heat and RSV.

We found that the function of Stvb-i paralogs is related to developmental homeostasis. The genes of this family are mainly expressed in immature tissues containing meristems, and their expression patterns differed at different growth stages. Stvb-i was specifically expressed in immature tissues of seedlings, tillers, and young panicles (Figure 4B). Its suppression inhibited plant growth for a long period from the vegetative to reproductive stages (Figures 5A,B and Supplementary Figure S5). The relationship between expression pattern and plant development was also observed for the Stvb-i-paralogous gene Os11g31500 (Figures 4B, 5C and Supplementary Figure S7). These results suggest that the $S t v b-i$ family genes affect plant growth and development. In particular, Stvb-i may support the growth of meristems. Stvb-i was highly expressed in calluses (Supplementary Figure S4), which are composed of cells capable of growth and division, similar to meristems.

In the seedling base of the resistant rice, the concentration and the spread of RSV were suppressed (Figure 1A). The mechanism of RSV resistance conferred by $S t v b-i$ may be similar to that employed to produce virus-free plants from an infected plant by meristem-tip culture (Spangenberg et al., 1998; Razdan, 2003). This technique is based on virus elimination from newly generated cells in meristems (Spangenberg et al., 1998), possibly because cell growth rate is higher than viral multiplication rate owing to vigorous metabolism of the host cells (Razdan, 2003). Stvb-i may help to maintain cell growth rate even in the meristems infected with RSV, reducing RSV concentration at every cell division. This would result in rice plant growth quickly escaping from spreading RSV.

$S t v b-i$ greatly affects the heat tolerance of plant growth. When exposed to high temperature, rice plants show various 


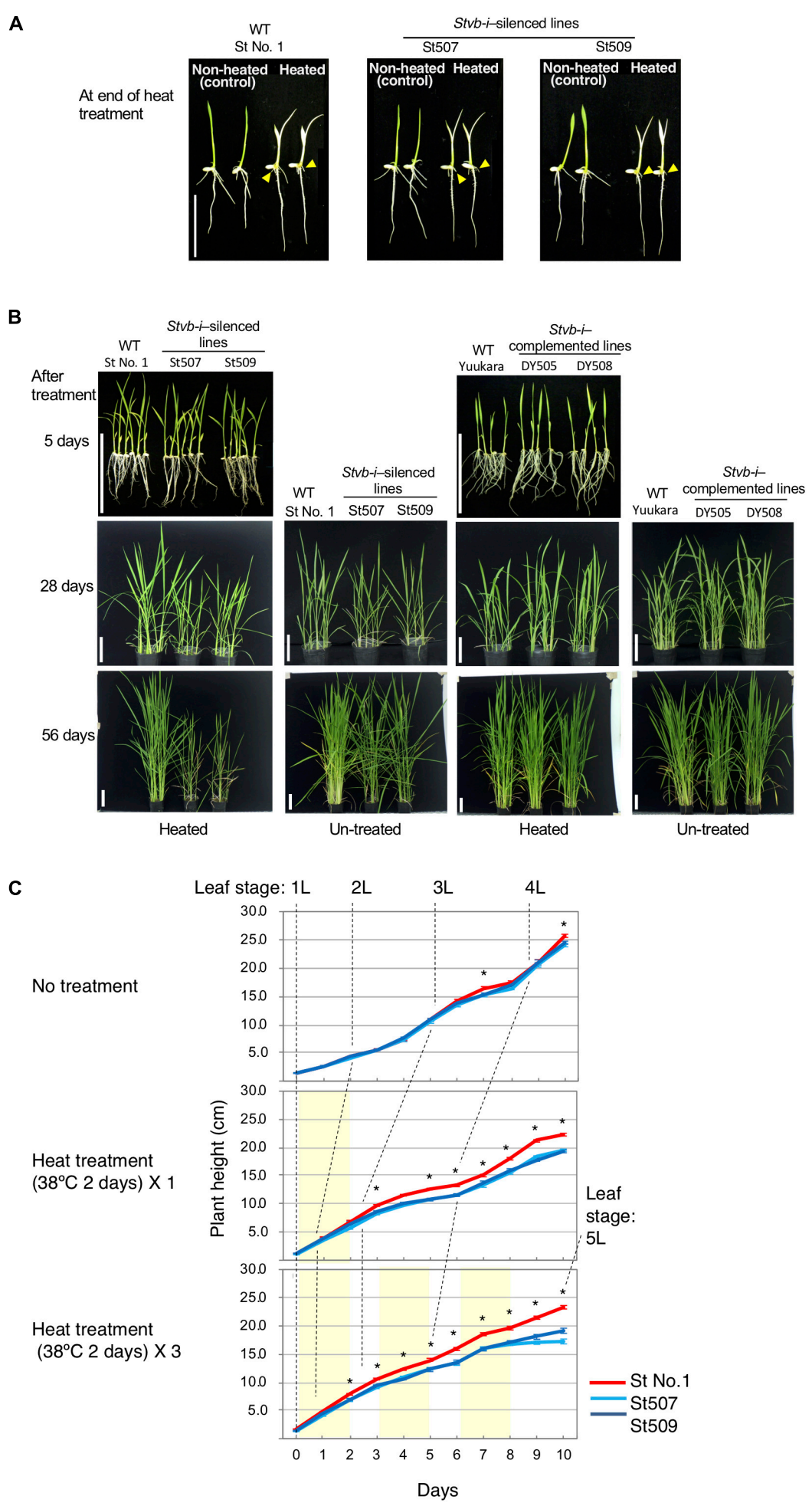

FIGURE 6 | Plant phenotypes of rice plant exposed to high temperature. (A) Seedlings of Stvb-i-silenced lines and the WT St No. 1 at the end of heat treatment at $35-37^{\circ} \mathrm{C}$ for 4 days at the 1st-leaf stage. Heat-treated plants exhibited chlorosis and short crown roots (yellow arrowheads), whereas non-heated seedlings did not. Scale bar: $3 \mathrm{~cm}$. (B) Appearance of Stvb-i-silenced plants and Stvb-i-complemented plants at 5, 28, and 56 days after the end of heat treatment (the same condition to panel A). Upper panel; representative heat-treated seedlings grown at $25^{\circ} \mathrm{C}$ in a growth chamber for 5 days after the end of the heat treatment. Middle and lower panels; plants grown for 28 and 56 days, respectively, after the heat treatment. Untreated plants were employed as controls for heated plants. Scale bars: $10 \mathrm{~cm}$. (C) Plant height of Stvb-i-silenced lines (St507 and St509) and the WT St No. 1 exposed to high temperature. Seedlings of at the 1st-leaf stage were left untreated or were treated at $38^{\circ} \mathrm{C}$ for 2 days once or three times. Light yellow shading represents heating periods. Asterisks show significant differences between both Stvb-i-silenced lines and WT (Tukey-Kramer test, $p<0.01, n=20$ ). 


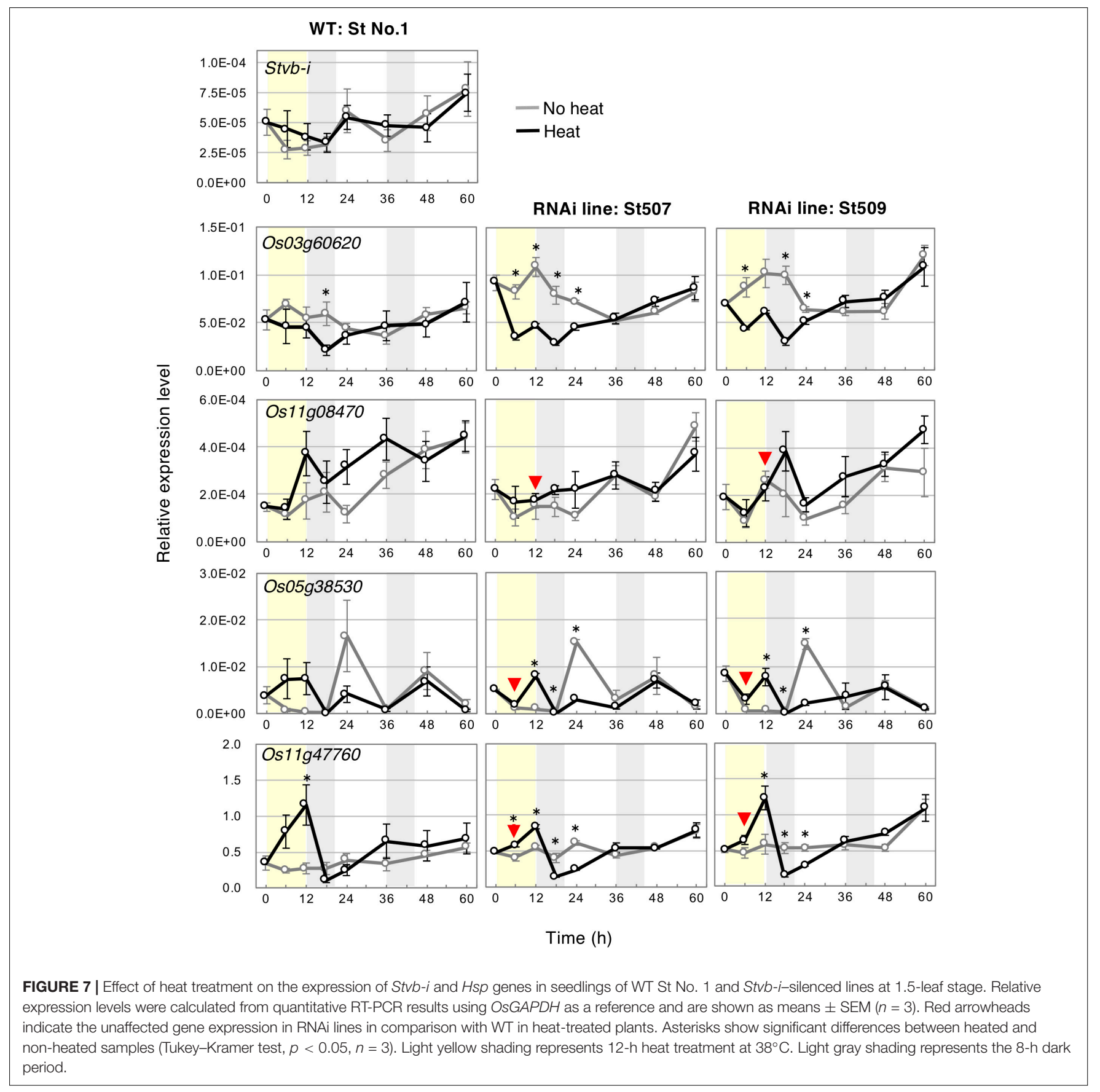

abnormal phenotypes: thin and short crown roots, chlorosis, wilting and stunting in seedlings, decrease in tiller number during vegetative growth, and defective panicle formation in the reproductive period (Krishnan et al., 2011). Similar abnormalities were observed at normal temperature in the $S t v b$-i-silenced plants (Figures 5A,B and Supplementary Figure S5A) and were exacerbated in plants exposed to high temperature for many days (Figure 6C and Supplementary Figure S5B). The role of Stvb-i in meristems is closely related to the response to heat stress.

Temperature above the optimum for growth impairs cell homeostasis (Bokszczanin et al., 2013), and plants regulate gene expression in response to heat (Liu et al., 2015). Heat shock proteins (Hsps) greatly contribute to cell homeostasis as molecular chaperones (Wang et al., 2004; Wahid et al., 2007). Hsp90s, a major Hsp family, regulate cell-cycle control, signaltransduction networks, and protein folding, trafficking, and degradation (Wang et al., 2004; Sangster and Queitsch, 2005). $S t v b-i$ is similar to Hsp90 in the structure of its $\mathrm{N}$-terminal domain, expression (constitutive under usual conditions), and function (contribution to recovery from heat stress and influence on plant development). Stvb-i may regulate heat-sensitive signaling cascades closely linked to meristem growth. 
RNA virus requires host Hsp70s (Mayer, 2005; Nagy et al., 2011). Hsp70s function together with Hsp90s as molecular chaperones, particularly in RNA metabolism (Walters and Parker, 2015; Jacob et al., 2017). In RSV infection, the involvement of rice Hsp70 genes, Os03g60620, Os11g08470, and Os05g38530, has been suggested (Jiang et al., 2014). Stvb-i silencing affected the expression of the Hsp70s (Figure 7 and Supplementary Figure S8) and growth of plant (Figures 5A,B), the results suggest a possibility that the four Hsp70s examined in this study function in plant development. The NbHsp70 (KX912913), homologous to Os03g60620, Os05g38530, and Os11g47760, is required for the normal growth and development of N. benthamiana (Yang et al., 2017). RSV may recruit Hsp70s for its multiplication and interfere with the native roles of Hsp70s, affecting rice meristem growth and causing systemic symptoms.

The RSV symptoms are similar to morphological changes in heat-damaged plants (Washio et al., 1967; Ling, 1972; Krishnan et al., 2011; Supplementary Figure S9). Growth stunting caused by RSV infection was significantly exacerbated by $S t v b$-i-silencing (Figure 5A). The results suggest that RSV multiplication may induce heat-like stress in meristematic cells and interfere with a cascade in which $S t v b-i$ is involved. Probably, the mechanism of meristem growth conferred by Stvb-i functions to alleviate not only stress caused by high temperature, but also stress caused by RSV.

The major type of plant disease resistance genes encodes NBS-LRR proteins, which confer resistance to pathogens by eliciting hypersensitive response (programmed cell death) and thus preventing pathogen invasion and propagation, which results in selective pressure. The interaction between an NBSLRR protein and pathogenic factor requires Hsp90 and is therefore temperature sensitive (Samuel, 1931; Kobayashi et al., 2014; Park and Seo, 2015; Qian et al., 2018). RSV resistance conferred by $S t v b-i$ has remained stable over the past 50 years despite an increase in the number of days when the temperature exceeded the optimal temperature for rice seedling growth, providing ideal conditions for RSV multiplication (Jiang et al., 2014; weather data are from the Japan Meteorological agency $\left.{ }^{16}\right)$. A stable expression of $S t v b-i$ (Figures 3B, 7), which contributes to such stress recovery of meristematic cells, will strongly and quickly suppress viral multiplication even under high pressures of RSV infection. Unlike in the case of the major resistance genes encoding NBS-LRR proteins, the viral resistance conferred by $S t v b-i$ is attributable to the basic response to heat stress in host meristematic cells. Although Stvb-i is unable to completely suppress RSV multiplication, it certainly confers durable and sustainable RSV tolerance combined with heat insensitivity.

Our study has demonstrated that rice acquires sustainable dual tolerance to abiotic and biotic stresses through the stability

${ }^{16} \mathrm{http} / / /$ www.data.jma.go.jp/

\section{REFERENCES}

Bokszczanin, K. L., Solanaceae Pollen Thermotolerance Initial Training Network Consortium, and Sotirios Fragkostefanakis (2013). Perspectives on deciphering mechanisms underlying plant heat stress response and thermotolerance. Front. Plant Sci. 4:315. doi: 10.3389/fpls.2013.00315 of plant growth. In the context of global warming, exploration of genes involved in the metabolic and signaling systems that maintain meristem growth will be conducive to conferring multitolerance in plants. Improvement of developmental stability will help secure the productivity of crops under diverse environmental conditions.

\section{DATA AVAILABILITY STATEMENT}

The datasets generated for this study are included in the article/Supplementary Materials.

\section{AUTHOR CONTRIBUTIONS}

YH-S and KH performed the experiments and analyzed the data. YH-S designed the experiments and wrote the manuscript.

\section{FUNDING}

This work was supported by grants from the Ministry of Agriculture, Forestry and Fisheries of Japan (Integrated Research Project for Plants, Insects, and Animals Using Genome Technology QT4006 and MP1107) and partially by JSPS KAKENHI Grant Number 16K07562.

\section{ACKNOWLEDGMENTS}

We are indebted to Drs. Naoki Sugiura and Toshihiko Izawa of Aichi Prefectural Agricultural Research Center for providing recombinant inbred line populations. We thank the Rice Genome Research Program, Japan, for providing the BAC library and for the sequence data, technical information, and support. We thank the RIKEN BioResource Research Center for providing the TAC vector pYLTAC7 and Prof. Ko Shimamoto (deceased) of Nara Technical University for providing the pANDA vector. We are indebted to Dr. Takayuki Mitsunaga of the National Agricultural Research Organization for statistical advice. We are indebted to Drs. Satoru Kashiwazaki (deceased), Mabito Iwasaki (deceased), and Koji Saito of the National Agricultural Research Organization for supporting this work.

\section{SUPPLEMENTARY MATERIAL}

The Supplementary Material for this article can be found online at: https://www.frontiersin.org/articles/10.3389/fpls.2020.00519/ full\#supplementary-material

Budot, B. O., Encabo, J. R., Ambita, I. D. V., Atienza-Grande, G. A., Satoh, K., Kondoh, H., et al. (2014). Suppression of cell wall-related genes associated with stunting of Oryza glaberrima infected with Rice tungro spherical virus. Front. Microbiol. 5:26. doi: 10.3389/fmicb.2014.00026

Hayano-Saito, Y. (2002). Coding strategy of Rice stripe virus and genetic analysis of rice stripe resistance. Bull. Natl. Agric. Res. Center Hokkaido Region 175, 1-45. 
Hayano-Saito, Y. (2014). Infection of Rice stripe virus in rice stripe-resistant cultivar harboring Stvb-i gene. Annu. Rep. Kanto Tosan Plant Protect. Soc. 61, 9-12. doi: 10.11337/ktpps.2014.9

Hayano-Saito, Y., Saito, K., Nakamura, S., Kawasaki, S., and Iwasaki, M. (2000). Fine physical mapping of the rice stripe resistance gene locus, Stvb-i. Theor. Appl. Genet. 101, 59-63. doi: 10.1007/s001220051449

Hayano-Saito, Y., Tsuji, T., Fujii, K., Saito, K., Iwasaki, M., and Saito, A. (1998). Localization of the rice stripe disease resistance gene, Stv-bi, by graphical genotyping and linkage analyses with molecular markers. Theor. Appl. Genet. 96, 1044-1049. doi: 10.1007/s001220050837

Hibino, H. (1996). Biology and epidemiology of rice viruses. Annu. Rev. Phytopathol. 34, 249-274. doi: 10.1146/annurev.phyto.34.1.249

Hoshikawa, K. (1989). The Growing Rice Plant. Tokyo: Nobunkyo.

Hu, W., Hu, G., and Han, B. (2009). Genome-wide survey and expression profiling of heat shock proteins and heat shock factors revealed overlapped and stress specific response under abiotic stress in rice. Plant Sci. 176, 583-590. doi: 10.1016/j.plantsci.2009.01.016

Jacob, P., Hirt, H., and Bendahmane, A. (2017). The heat-shock protein/chaperone network and multiple stress resistance. Plant Biotechnol. J. 15, 405-414. doi: 10.1111/pbi.12659

Jiang, S., Lu, Y., Li, K., Lin, L., Zheng, H., Yan, F., et al. (2014). Heat shock protein 70 is necessary for Rice stripe virus infection in plants. Mol. Plant Pathol. 15, 907-917. doi: 10.1111/mpp.12153

Kawahara, Y., Bastide, M., Hamilton, J. P., Kanamori, H., McCombie, W. R., Ouyang, S., et al. (2013). Improvement of the Oryza sativa Nipponbare reference genome using next generation sequence and optical map data. Rice 6:4. doi: 10.1186/1939-8433-6-4

Kobayashi, K., Sekine, K., and Nishiguchi, M. (2014). Breakdown of plant virus resistance: can we predict and extent the durability of virus resistance? J. Gen. Plant Pathol. 80, 327-336. doi: 10.1007/s10327-014-0527-1

Koumura, T., Takamatsu, M., Haga, T., Syaku, I., Suymiya, A., Tanabe, K., et al. (1980). A new rice variety "Koganebare". Res. Bill. Aichi Agric. Res. Cent. 12, $1-13$.

Krishnan, P., Ramakrishnan, B., Raja Reddy, K., and Reddy, V. R. (2011). Hightemperature effects on rice growth, yield, and grain quality. Adv. Agron. 111, 87-206. doi: 10.1016/B978-0-12-387689-8.00004-7

Kwon, T., Lee, J. H., Park, S. K., Hwang, U.-H., Cho, J.-H., Kwak, D.-Y., et al. (2012). Fine mapping and identification candidate rice genes associated with $q S T V 11^{S G}$, a major QTL for rice stripe disease resistance. Theor. Appl. Genet. 125, 1033-1046. doi: 10.1007/s00122-012-1893-8

Ling, K. C. (1972). Rice Virus Diseases. Los Baños: The International Rice Research Institute.

Liu, J., Feng, L., Li, J., and He, Z. (2015). Genetic and epigenetic control of plant heat responses. Front. Plant Sci. 6:267. doi: 10.3389/fpls.2015.00267

Liu, Y. G., Shirano, Y., Fukai, H., Yanai, Y., Tasaka, M., Tabata, S., et al. (1999). Complementation of plant mutants with large genomic DNA fragments by transformation-competent artificial chromosome vector accelerates positional cloning. Proc. Natl. Acad. Sci. U.S.A. 96, 6535-6540. doi: 10.1073/pnas.96.11. 6535

Maeda, H., Matsushita, K., Iida, S., and Sunohara, Y. (2006). Characterization of two QTLs controlling resistance to rice stripe virus detected in a Japanese upland rice line, Kanto 72. Breed. Sci. 56, 359-364. doi: 10.1270/jsbbs.56.359

Mayer, M. P. (2005). Recruitment of Hsp70 chaperons: a crucial part of viral survival strategies. Rev. Physiol. Biochem. Pharmacol. 153, 1-46. doi: 10.1007/ s10254-004-0025-5

Miki, D., and Shimamoto, K. (2004). Simple RNAi vector for stable and transient suppression of gene function in rice. Plant Cell Physiol. 45, 490-495. doi: 10. 1093/pcp/pch048

Nagy, P. D., Wang, R. Y., Pogany, J., Hafren, A., and Makinen, K. (2011). Emerging picture of host chaperone and cyclophilin roles in RNA virus replication. Virology 411, 374-382. doi: 10.1016/j.virol.2010.12.061

Otuka, A. (2013). Migration of rice planthoppers and their vectored re-emerging and novel rice viruses in East Asia. Front. Microbiol. 4:309. doi: 10.3389/fmicb. 2013.00309

Ozawa, K. (2009). Establishment of a high efficiency Agrobacterium-mediated transformation system of rice (Oryza sativa L.). Plant Sci. 176, 522-527. doi: 10.1016/j.plantsci.2009.01.013
Park, C. J., and Seo, Y. S. (2015). Heat shock proteins: a review of the molecular chaperones for plant immunity. Plant Pathol. J. 31, 323-333. doi: 10.5423/PPJ. RW.08.2015.0150

Qian, L., Zhao, J., Du, Y., Zhao, X., Han, M., and Liu, T. (2018). Hsp90 interacts with Tm- $2^{2}$ and is essential for Tm- $2^{2}$-mediated resistance to Tobacco mosaic virus. Front. Plant Sci. 9:411. doi: 10.3389/fpls.2018.00411

Razdan, M. K. (2003). Introduction to Plant Tissue Culture, 2nd Edn, New Hampshire: Science Publisher.

Saito, K., Hayano-Saito, Y., Kuroki, M., and Sato, Y. (2010). Map-based cloning of the rice cold tolerance gene Ctb1. Plant Sci. 179, 97-102. doi: 10.1016/j.plantsci. 2010.04.004

Sakata, K., Nagamura, Y., Numa, H., Nagasaki, H., Idonuma, A., Watanabe, W., et al. (2002). RiceGAAS: an automated annotation system and database for rice genome sequence. Nucleic Acids Res. 30, 98-102. doi: 10.1093/nar/30.1.98

Samuel, G. (1931). Some experiments on inoculating methods with plant viruses, and on local lesions. Ann. Appl. Biol. 18, 494-507.

Sangster, T. A., and Queitsch, C. (2005). The HSP90 chaperone complex, an emerging force in plant development and phenotypic plasticity. Curr. Opin. Plant Biol. 8, 86-92. doi: 10.1016/j.pbi.2004.11.012

Satoh, K., Kondoh, H., Sasaya, T., Shimizu, T., Choi, I. R., Omura, T., et al. (2010). Selective modification of rice (Oryza sativa) gene expression by rice stripe virus infection. J. Gen. Virol. 91, 294-305. doi: 10.1099/vir.0.015990-0

Satoh, K., Shimizu, T., Kondoh, H., Hiraguri, A., Sasaya, T., Choi, I. R., et al. (2011). Relationship between symptoms and gene expression induced by the infection of three strains of Rice dwarf virus. PLoS One 6:e18094. doi: 10.1371/journal. pone.0018094

Satoh, K., Yoneyama, K., Kondoh, H., Shimizu, T., Sasaya, T., Choi, I. R., et al. (2013). Relationship between gene responses and symptoms induced by Rice grassy stunt virus. Front. Microbiol. 4:313. doi: 10.3389/fmicb.2013. 00313

Sonku, Y., and Sakurai, Y. (1973). Transmission of Rice stripe virus by Laodelphax striatellus (Fallén) II. Translocation of the hopper-secreted virus and location of virus multiplication within a rice plant. Ann. Phytopathol. Soc. Jpn. 39, 109-119.

Spangenberg, G., Wang, Z. Y., and Potrykus, I. (1998). "Meristem culture," in Biotechnology in Forage and Turf Grass Improvement. Monographs on Theoretical and Applied Genetics, Vol. 23, eds. R. Frankel, M. Grossman, H. F. Linskens, P. Maliga, and R. Riley (Berlin: Springer), 7-17.

Takahashi, M., Ishikawa, K., Hayano-Saito, Y., and Tsuchiya, K. (2008). Multiplication of Rice stripe virus in the shoot apex is the first step toward systemic spread. The 9th international congress of plant pathology, 2008 Aug 24-29; Trino, Italy. J. Plant Pathol. 90:245.

Takahashi, M., Toriyama, S., Hamamatsu, C., and Ishihama, A. (1993). Nucleotide sequence and possible ambisense coding strategy of rice stripe virus RNA segment 2. J. Gen. Virol. 74, 769-773. doi: 10.1099/0022-1317-74-4-769

Toriyama, S., Takahashi, M., Sano, Y., Shimizu, T., and Ishihama, A. (1994). Nucleotide sequence of RNA1, the largest genomic segment of rice stripe virus, the prototype of the tenuiviruses. J. Gen. Virol. 75, 3569-3579. doi: 10.1099/ 0022-1317-75-12-3569

Wahid, A., Gelani, S., Ashraf, M., and Foolad, M. R. (2007). Heat tolerance in plants: an overview. Environ. Exp. Bot. 61, 199-223. doi: 10.1016/j.envexpbot. 2007.05.011

Walters, R. W., and Parker, R. (2015). Coupling of ribostasis and proteostasis: Hsp70 proteins in mRNA metabolism. Trends Biochem. Sci. 40, 552-559. doi: 10.1016/j.tibs.2015.08.004

Wang, B., Jiang, L., Zhang, Y., Zhang, W., Wang, Q., Liu, S., et al. (2011). Genetic dissection of the resistance to Rice stripe virus present in the indica rice cultivar 'IR24'. Genome 54, 611-619. doi: 10.1139/g11-022

Wang, G. D., Tian, P. F., Cheng, Z. K., Wu, G., Jiang, J. M., Li, D. B., et al. (2003). Genomic characterization of Rim2/Hipa elements reveals a CACTA-like transposon superfamily with unique features in the rice genome. Mol. Genet. Genomics 270, 234-242. doi: 10.1007/s00438-003-0918-Z

Wang, Q., Liu, Y., He, J., Zheng, X., Hu, J., Liu, Y., et al. (2014). STV11 encodes a sulphotranseferase and confers durable resistance to rice stripe virus. Nat. Commun. 5:4768. doi: 10.1038/ncomms5768

Wang, W., Vinocur, B., Shoseyov, O., and Altman, A. (2004). Role of plant heatshock proteins and molecular chaperones in the abiotic stress response. Trends Plant Sci. 9, 244-252. doi: 10.1016/j.tplants.2004.03.006 
Wang, Y., Bao, Z., Zhu, Y., and Hua, J. (2009). Analysis of temperature modulation of plant defense against biotrophic microbes. Mol. Plant Microbe Interact. 22, 498-506. doi: 10.1094/MPMI-22-5-0498

Washio, O., Ezuka, A., Sakurai, Y., and Toriyama, K. (1967). Studies on the breeding of rice varieties resistant to stripe disease. I. Varietal difference in resistance to stripe disease. Jpn. J. Breed. 17, 19-26.

Washio, O., Ezuka, A., Sakurai, Y., and Toriyama, K. (1968). Testing method for, genetics of and breeding for resistance to rice stripe disease. Bull. Chugoku Natl. Agric. Exp. Stat. A 16, 39-197.

Wu, X., Zuo, S., Chen, Z., Zhang, Y., Zhu, J., Ma, N., et al. (2011). Fine mapping of $q S T V 11^{T Q}$, a major gene conferring resistance to rice stripe disease. Theor. Appl. Genet. 122, 915-923. doi: 10.1007/s00122-0101498-z

Xiong, R., Wu, J., Zhou, Y., and Zhou, X. (2009). Characterization and subcellular localization of an RNA silencing suppressor encoded by Rice stripe tenuivirus. Virology 387, 29-40. doi: 10.1016/j.virol.2009.01.045

Yang, J., Zhang, F. Z., Cai, N. J., Wu, N., Chen, X., Li, J., et al. (2017). A furoviral replicase recruits host HSP70 to membranes for viral RNA replication. Sci. Rep. 7:45590. doi: 10.1038/srep45590

Zhang, Y. X., Wang, Q., Jiang, L., Liu, L. L., Wang, B. X., Shen, Y. Y., et al. (2011). Fine mapping of $q S T V 11^{K A S}$, a major QTL for rice stripe disease resistance. Theor. Appl. Genet. 122, 1591-1604. doi: 10.1007/s00122-0111557-0

Zhu, Y., Hayakawa, T., and Toriyama, S. (1992). Complete nucleotide sequence of RNA 4 of rice stripe virus isolate $\mathrm{T}$, and comparison with another isolate and with maize stripe virus. J. Gen. Virol. 73, 1309-1312. doi: 10.1099/0022-131773-5-1309

Zhu, Y., Hayakawa, T., Toriyama, S., and Takahashi, M. (1991). Complete nucleotide sequence of RNA 3 of rice stripe virus: an ambisense coding strategy. J. Gen. Virol. 72, 763-767. doi: 10.1099/0022-1317-72$4-763$

Conflict of Interest: The authors declare that the research was conducted in the absence of any commercial or financial relationships that could be construed as a potential conflict of interest.

Copyright (c) 2020 Hayano-Saito and Hayashi. This is an open-access article distributed under the terms of the Creative Commons Attribution License (CC BY). The use, distribution or reproduction in other forums is permitted, provided the original author(s) and the copyright owner(s) are credited and that the original publication in this journal is cited, in accordance with accepted academic practice. No use, distribution or reproduction is permitted which does not comply with these terms. 